\title{
La matérialité : une ressource pour l'enseignement-apprentissage de la lecture
}

The materiality: a resource for teaching-learning to read

\section{Claire Taisson-Perdicakis}

\section{(2) OpenEdition}

\section{Journals}

Édition électronique

URL : http://journals.openedition.org/educationdidactique/1984

DOI : 10.4000/educationdidactique.1984

ISSN : 2111-4838

Éditeur

Presses universitaires de Rennes

\section{Édition imprimée}

Date de publication : 12 décembre 2014

Pagination : 125-138

ISBN : 978-2-7535-39-66-2

ISSN : 1956-3485

\section{Référence électronique}

Claire Taisson-Perdicakis, «La matérialité : une ressource pour l'enseignement-apprentissage de la lecture », Éducation et didactique [En ligne], 8-2 | 2014, mis en ligne le 12 décembre 2016, consulté le 19 avril 2019. URL : http://journals.openedition.org/educationdidactique/1984 ; DOI : 10.4000/ educationdidactique.1984 


\title{
LA MATÉRIALITÉ : UNE RESSOURCE POUR L'ENSEIGNEMENT-APPRENTISSAGE DE LA LECTURE
}

\author{
Claire Taisson-Perdicakis
}

Universités de Lausanne et de Lorraine

\begin{abstract}
Larticle qui suit est issu d'une recherche longitudinale pour laquelle une cohorte d'élèves a été suivie durant tout le cycle 2 dans des situations ordinaires de classe. Au cycle 2, l'un des principaux objectifs des programmes scolaires est l'apprentissage de la lecture. Les fondations théoriques sont le paradigme vygotskien et la didactique de la lecture. Larticle expose comment les élèves construisent leur savoir-lire dans les dimensions code et sens, au moyen de la matérialité envisagée comme ressource pour l'enseignement-apprentissage de la lecture.
\end{abstract}

Mots-clés : Enseignement-apprentissage, Matérialité, Didactique de la lecture, Paradigme historico-culturel, Développement psychologique de l'enfant.

\section{The materiality : a resource for teaching-learning to read}

This paper comes from a research which consists of a longitudinal study in which one group of pupils in ordinary classroom situations has been followed from the top level of pre-elementary school to the second level of primary school. For these three school grades, one of the main objectives in the curriculum is learning to read. The epistemological framework is the cultural-historical psychology and didactics of reading. Pupils build their new reading skills, i.e. code and meaning, by resorting to the materiality which appears as a resource for teaching-learning to read.

Keywords: Teaching-learning, Didactics of the reading, Materiality, Cultural-historical psychology, Child's psychological development. 


\section{INTRODUCTION}

L'enseignement-apprentissage de la lecture à l'école est depuis longtemps un sujet polémique: les médias, les ministres successifs de l'Éducation nationale, la vox populi... invoquent souvent le taux d'élèves qui entrent en sixième ne maîtrisant pas la lecture. Allègre, ministre de l'Éducation nationale de 1997 à 2000, a pu ainsi écrire : « Nombre d'élèves entrent en sixième sans savoir lire ni écrire correctement » (2007, p. 8). Pendant l'année scolaire 20062007, au sein même de la profession enseignante, l'enseignement-apprentissage de la lecture a été au centre d'âpres discussions. En effet, en accordant la priorité à l'enseignement du code alphabétique, la circulaire ministérielle de 2006 a réveillé le débat sur les «méthodes de lecture ». La question de l'apprentissage de la lecture et de ses méthodes apparaît donc comme une préoccupation centrale et toujours actuelle pour la formation des élèves.

Mais qu'entend-on par "méthode de lecture »? Pour le grand public, elle consiste généralement en l'application de principes contenus dans un manuel de Cours Préparatoire. Cette conception transparaît aussi dans les programmes ministériels qui exhortent à recourir à " un manuel scolaire de qualité » (2006a, p. 70). Pour le chercheur spécialiste de l'enseignement-apprentissage de la lecture envisagé comme processus complexe, la méthode est définie par « les principes qui sous-tendent l'action des maîtres en charge des premiers apprentissages de la langue écrite » (Goigoux, 2008b, p. 444). Les méthodes de lecture diffèrent ainsi en fonction de six critères (Goigoux, 2003) : les relations entre l'oral et l'écrit, la primeur du signe ou du sens, l'ordre d'étude des différentes unités linguistiques, les modalités d'identification des mots en contexte, l'importance accordée à la production de mots et de textes, la place de l'enseignement de la compréhension de textes et l'acculturation. Cependant, selon la définition supra, ces critères ne font que « soustendre l'action des maîtres ». En ce sens, Nonnon et Goigoux expliquent : « Le travail mené par les enseignants et les élèves dans les tâches de lecture n'est pas une simple application d'un modèle didactique a priori, d'où découleraient de façon univoque les pratiques et leurs effets sur l'apprentissage. [...] Des chercheurs ont signalé que la référence classique aux méthodes de lecture n'était pas le niveau pertinent pour analyser la façon dont est mis en œuvre l'enseignement de la lecture $[\ldots] »(2008$, p. 6-7).
Dans cet article nous suivons ces auteurs et proposons de nous attacher à l'activité effective dans les pratiques réelles d'enseignement-apprentissage de la lecture. Car « toutes les pratiques sont singulières »(Goigoux, 2003, p. 20). Des enseignants peuvent utiliser la même méthode ou encore le même manuel, pour autant leurs pratiques s'avérer profondément différentes (Nonnon \& Goigoux, 2008). Nous menons aussi nos investigations au-delà du cadre strict du Cours Préparatoire pour les inscrire dans un empan temporel élargi au cycle 2 (Grande Section - Cours Préparatoire - Cours Élémentaire 1), trois années considérées comme la période de l'apprentissage de la lecture par excellence.

Parmi les éléments singuliers observables dans les situations d'enseignement-apprentissage de la lecture au cycle 2 , nous mettons en évidence l'importance de la matérialité en effectuant une étude de cas pour laquelle une cohorte d'élèves avec l'enseignante de la classe est suivie durant les trois années du cycle dans une classe multiniveaux regroupant la Grande Section, le Cours Préparatoire et le Cours Élémentaire $1^{\text {re }}$ année. La recherche dont notre article est issu tente de rendre compte du rôle de la matérialité lors de séances d'enseignement-apprentissage de la lecture dans cette classe. La première section propose un état des lieux de la didactique de la lecture permettant de situer la pratique de l'enseignante observée. La deuxième section s'intéresse au concept de matérialité. Dans la troisième section, des exemples illustrent comment se réalise in situ l'enseignement-apprentissage de la lecture au moyen d'outils matériels divers.

\section{LA DIDACTIQUE DE LA LECTURE, ÉTAT DES LIEUX}

\section{Les dimensions de la lecture}

La lecture est « un processus complexe qui consiste à mettre en relation des symboles orthographiques avec leur son (phonologie) et leur signification (sémantique) » (Ziegler, 2008, p. 440). Lire c'est à la fois identifier des mots transcrits alphabétiquement et extraire la signification d'un écrit dans son entièreté. La lecture porte ainsi deux dimensions phares, le code et le sens. Historiquement, ces deux grandes dimensions ont souvent été opposées. L'apprentissage du déchiffrage du code alphabétique a longtemps été privilégié mais, à partir des années 
1970, parce que les élèves sont destinés à suivre des études secondaires, savoir déchiffrer ne suffit plus. C'est alors l'apprentissage de la compréhension qui domine la didactique de la lecture (Goigoux, 2003).

Aujourd'hui, les recherches en didactique et en neurosciences apportent un certain nombre de points d'appui scientifiques à l'enseignement-apprentissage de la lecture. Selon Goigoux l'activité d'identification des mots est la première à être engagée dans l'activité de compréhension. Concernant cette activité d'identification des mots, les neurosciences, notamment les recherches par imagerie médicale, viennent éclairer le fonctionnement du cerveau qui lit. Dehaene (2007) considère en effet qu'est advenue une science de la lecture selon laquelle lire implique de développer une connexion efficace entre la vision des lettres et le codage des sons du langage, et d'apprendre les correspondances entre graphèmes et phonèmes. L'identification des mots procède de deux voies. La voie indirecte est mise en action face à un mot écrit jamais rencontré par le lecteur. Elle consiste en quatre phases : le mot écrit est déchiffré ; ce mot écrit déchiffré est ensuite oralisé donnant une image auditive ; cette image auditive est signifiante si le mot appartient au lexique oral de l'élève qui accède alors à la compréhension du mot écrit. La voie directe est celle qui est mise en action face à un mot écrit déjà rencontré et stocké dans la mémoire du lecteur. Elle consiste en trois phases : le mot écrit est instantanément reconnu car il est déjà en mémoire sous forme d'image orthographique; cette image orthographique est signifiante car le mot appartient au lexique du lecteur ; ce mot quasi instantanément reconnu est compris. «La compréhension passe avant tout par la fluidité du décodage» (p. 301). Cette fluidité caractérise l'identification des mots par voie directe qui est le résultat d'un entraînement de la voie indirecte ou déchiffrage. L'identification des mots par la voie directe n'est rendue possible que par l'automatisation du processus indirect à force de rencontres nombreuses avec les mots.

Goigoux (2008a) estime essentiel et incontournable le processus d'identification des mots que nous venons d'expliciter. Cependant il le replace dans le cadre plus large de toutes les compétences à acquérir pour apprendre à lire. Le processus d'identification des mots participe de la compréhension, à laquelle toutefois il ne suffit pas. En effet la compréhension d'un texte écrit ne résulte pas de la seule automatisation de l'identification des mots après laquelle le sens du texte se donnerait de manière irrépressible ; elle est une activité spécifique qui doit faire l'objet d'un apprentissage structuré. La compréhension du texte écrit est le résultat de l'exercice des trois activités d'identification, linguistique, mentale, simultanées et en interaction. L'activité linguistique requiert des connaissances lexicales et syntaxiques et consiste à relier les significations pour aller jusqu'à l'idée portée par les groupes de mots. L'activité mentale a pour objet la construction de la représentation mentale de la situation évoquée par le texte qui suppose la capacité du lecteur à faire des inférences, c'est-à-dire des opérations logiques de déduction rendant explicite, à partir d'indices présents dans le texte, une information qui n'est qu'évoquée ou supposée connue. Le lecteur "se fait un film dans sa tête ». Cet exercice s'enseigne de manière explicite, et s'apprend, car il nécessite l'acquisition de compétences stratégiques d'autorégulation comme le contrôle, l'évaluation et la régulation de sa propre activité de lecture.

\section{Apprendre à lire au cycle 2}

Les deux grandes dimensions de l'acte de lire, identification des mots et compréhension, doivent donc être enseignées. Goigoux (2003) légitime à cet effet les conceptions dites "interactives " qui font intervenir les connaissances sémantiques, syntaxiques, orthographiques, alphabétiques, phonologiques, du lecteur. " Dans les méthodologies interactives, la "leçon de lecture » est avant tout une lecture collective de texte. Elle met en scène, sous la tutelle du maître, les activités cognitives complexes qui constituent l'acte de lecture afin qu'elles soient progressivement reconstruites puis intériorisées par chaque élève. En d'autres termes, le maître incite à réaliser successivement, lentement et collectivement, les actions que les élèves devront ensuite mettre en œuvre simultanément, rapidement et individuellement $»($ p. 15).

L'enseignante observée dispense ce type d'enseignement. Dès la Grande Section, elle propose aux élèves des écrits authentiques longs qui dépassent leur capacité de lecture autonome. L'étude de ces textes aux trois niveaux donne à voir un travail simultané du code, du sens et des genres (affiche, recette, épisodes d'album, documentaires provenant d'Internet, textes du patrimoine...) ; et la production d'écrit est régulière, sous forme de dictée à l'adulte 
en Grande Section. Au Cours Préparatoire et au Cours Élémentaire $1^{\text {re }}$ année est utilisée la méthode Mika (méthode interactive de lecture élaborée par Chauveau, 1997 chez Retz) complétée par d'autres sources textuelles. Le domaine de la phonologie est quant à lui traité dans des séances spécifiques dont l'objectif est explicité aux élèves.

\section{UN LEVIER PUISSANT POUR FAIRE APPRENDRE À LIRE : LA MATÉRIALITÉ}

\section{Perspective socio-culturelle}

L'étude menée s'inscrit dans le paradigme socioculturel et sémiotique du développement selon Vygotski. Dans ce cadre, l'écrit est au langage ce que l'algèbre est à l'arithmétique (1934/1997). Accéder à l'écrit c'est faire une utilisation abstraite du langage. L'enfant passe d'une matérialité sonore à une matérialité écrite, d'une chair à une autre chair. Outre cela, il est soumis à la double abstraction, celle de l'aspect sonore du langage et celle de l'interlocuteur. C'est pourquoi la lecture est un objet à enseigner. Car, explique Vygotski (1933/2012a, p. 162), « [...] la lecture n'est pas la simple association entre des signes graphiques et les sons qui y correspondent. La lecture est un processus complexe auquel les fonctions psychiques supérieures en ce qui concerne la pensée participent directement ». Autrement dit la lecture n'est pas seulement une traduction graphophonique ou déchiffrage mais implique aussi la mise en jeu du raisonnement. "La compréhension du récit est similaire à la résolution d'un problème en mathématique » (p. 164). En comprenant la lecture comme un objet composé de deux dimensions, à savoir déchiffrer et comprendre, Vygotski se place délibérément dans une forme d'apprentissage réflexif, non mécaniste, appelant des capacités à résoudre un problème. Il l'inclut dans le groupe des fonctions psychiques supérieures dont la formation caractérise le développement intellectuel de l'élève.

Le processus de formation des fonctions psychiques supérieures, dont la lecture, requiert l'intervention de l'adulte par l'acte d'enseignement sur la scène sociale scolaire ${ }^{1}$. Pris dans son développement actuel constitué de son déjà-là et déterminé à l'aide de problèmes résolus seul (Vygotski, 1933/2012b), l'élève est appelé à fonctionner dans un contexte en rupture avec ce déjà-là. Dans la classe s'instaure donc une dialectique entre les capacités des élèves qui constituent leur développement actuel, et les objectifs des programmes scolaires qui caractérisent leur développement potentiel, lequel est déterminé à l'aide de problèmes résolus sous la direction de l'enseignant ou en coopération avec des camarades plus avancés que lui (ibid.). « Le rôle de l'enseignement dans le développement de l'enfant consiste dans le fait que l'apprentissage crée la zone de développement le plus proche " (1933/2012a, p. 171). Cette zone de développement le plus proche ou zone proximale de développement correspond à l'écart entre développement actuel et développement potentiel (1933/2012b). L'enjeu de notre recherche est donc de montrer comment joue, au travers de la zone proximale de développement, la dialectique entre le déjà-là des élèves et les nouveaux savoirs en lecture contenus dans le programme scolaire. Nous observons et analysons comment l'enseignante initie un premier rapport aux compétences de lecture caractérisant le développement potentiel des élèves.

\section{Prolongement du paradigme socio-culturel}

L'œuvre de Vygotski est dominée par la prééminence du langage pour le développement intellectuel. Or Moro et Rodríguez (2005) élargissent la conception vygotskienne du développement en montrant que le bébé, parce qu'il vit dans un environnement social et culturel, s'approprie les usages canoniques et symboliques des objets. L'objet matériel, dont l'usage est signifié par l'adulte, au sein de la triade adulte-bébé-objet apparaît comme source du développement de l'enfant. Il s'ensuit que « [...] les conduites humaines sont fondamentalement fruit de la culture, et en ce sens résultent de significations publiques et ce, dès leurs prémices, i.e. avant l'avènement du langage » (Moro \& Rodríguez, 2005, p. 415). L'objet matériel, dont l'usage est médiatisé par l'adulte, joue donc un rôle capital dans le développement de l'enfant dès la naissance. D'autres études (Dimitrova, 2012 ; Moro, 2013 ; Tapparel, 2013) mettent en évidence le rôle de l'objet matériel dans le développement psychologique dans la prime enfance, en centre de vie enfantine lausannois et à l'école maternelle française.

À la suite de ces travaux, nous convoquons le concept de matérialité décliné selon deux modalités (Moro \& Joannes, à paraître) : d'une part la 
matérialité objectale qui tient à l'organisation de l'espace, aux objets et leurs usages, d'autre part la corporéité/gestualité qui concerne les gestes, les postures, les mimiques, la prosodie mais aussi la proxémie $^{2}$ (Forest, 2006). Pour notre recherche sur l'enseignement-apprentissage de la lecture, nous qualifions la matérialité au sens de Moro et Joannes de matérialité extrinsèque, par opposition à la matérialité intrinsèque définie comme l'ensemble des signes graphiques constitutifs du français écrit. La matérialité intrinsèque de l'écrit est pour partie le fait du système alphabétique qui est une des bases (mais pas l'unique base) de son orthographe. Catach (1978/2003) décrit le plurisystème du français écrit. « Notre « plurisystème » est une orthographe phonologique (à plus de $80 \%$ ), dans une certaine mesure morphologique (avec une concentration extrême des « morphogrammes grammaticaux », bien moindre des « morphogrammes lexicaux »), et enfin en partie distinctive » (p. 66). En résumé, l'ensemble des six codes (Chauveau, 1998) - alphabétique dont phonographique, morphographique, logographique/extraalphabétiques dont typographique, des supports, textuel - constitutifs du français écrit, peut être désigné comme la matérialité intrinsèque de l'écrit. Le rapport entre les deux matérialités ainsi définies est le suivant : la matérialité extrinsèque correspond à l'ensemble des outils intermédiaires auxiliaires dont se sert l'enseignant pour faire accéder l'élève à la matérialité intrinsèque de l'écrit.

\section{UNE ÉTUDE DE CAS COMME ILLUSTRATION}

\section{Méthodologie employée}

La méthodologie à caractère ethnographique (Cefaï, 2010) consiste en une démarche d'enquête qui s'appuie d'abord sur un recueil fractionné d'observations filmées de situations ordinaires de classe. Ainsi, une cohorte d'élèves est suivie in situ durant les trois années du cycle 2 de 2007 à 2010 au moyen d'un dispositif longitudinal : les douze observations filmées des séances entières de lecture ont lieu tout au long des trois années du cycle selon un rythme régulier de quatre fois par année scolaire. Deux entretiens semi-directifs avec l'enseignante permettent d'éclairer l'interprétation des données filmées. Dans le cadre d'une méthodologie ethnographique, l'entretien, en complément d'observations directes, prend en effet un caractère informatif et descriptif prononcé (Blanchet \& Gotman, 2001). Nous réalisons enfin un entretien d'auto-confrontation occasionnant, lors du visionnement chronologique d'extraits vidéos sélectionnés, une situation de dialogue chercheuse/ enseignante (Goigoux, 2002), orienté par un guide de questionnement pensé au préalable et focalisé sur la corporéité.

Les données filmées sont réduites sous la forme de synopsis dont la définition est empruntée au GRAFE, Groupe Romand d'Analyse du Français Enseigné de l'université de Genève. Les synopsis permettent d'extraire des informations essentielles et de procéder à la reformulation/réduction des éléments retenus en fonction des objectifs de la recherche (Blaser, 2009). Ces éléments retenus reformulés et réduits constituent les épisodes significatifs, moments à forte densité didactique (i.e. prometteuse d'une transformation des savoirs des élèves), porteurs d'un condensé d'interactions verbales et non verbales, et caractérisés par la présence ou l'« absence » de matérialité extrinsèque eu égard au contenu de savoir. Les épisodes significatifs sont "découpés » en événements remarquables d'empan temporel plus court facilitant donc les analyses microgénétiques.

Deux outils sont conçus pour analyser la matérialité au sein des événements remarquables : le tableau des matérialités et le photogramme. Chaque tableau des matérialités se rapporte à un épisode significatif dont il inventorie tous les éléments matériels, de nature intrinsèque ou extrinsèque. Chaque photogramme se rapporte à un événement remarquable dont il tend à représenter la dynamique de l'action en englobant captures d'images et corporéité, discours, description de l'action et fonction des gestes. Les éléments du photogramme sont de nature différente. La première colonne porte l'image au caractère analogique (parfois réhaussée de signes graphiques comme des flèches... permettant de traduire l'aspect dynamique du geste), accompagnée d'une description digitale succincte des gestes (Sensevy, 2011); la deuxième colonne présente la transcription du discours des protagonistes; la troisième colonne donne la description narrée de l'action; la quatrième colonne est l'interprétation de la matérialité gestuelle déployée. La compréhension de ce qui se joue dans la situation, la signification de l'événement remarquable, ressortent des représentations et des commentaires associés, lesquels font système (ibid.). 


\section{Exemples}

En préalable, l'apprentissage de la lecture nécessite l'exercice de l'attention. L'organisation de l'espace et les formes sociales de travail qui en résultent sont engagées dans ce processus. Les configurations spatiales sont variées et accueillent tout au long du cycle les élèves en petit groupe autour d'un support collectif ${ }^{3}$. La distance personnelle (45 à $135 \mathrm{~cm}$ ), telle que définie par la proxémique (Hall, 1966/1971), facilite le maintien de l'attention parce qu'elle permet à l'enseignante, d'un simple geste et de pointages, de recentrer son public sur l'objet de savoir. Par ailleurs le support collectif est à portée d'yeux et de mains des protagonistes favorisant les touchers directs, les tracés et les manipulations.

\section{$1^{\text {er }}$ exemple : la matérialité comme ressource pour} enseigner-apprendre les codes écrits

Voici un événement remarquable pris sur la deuxième séance filmée de Grande Section ( $2^{\mathrm{e}}$ séance filmée de l'année scolaire et du recueil complet) dont l'objectif est de faire lire la recette des crêpes. Le support de lecture est en lien avec la Chandeleur fêtée dans la classe. Les élèves feront les crêpes ultérieurement ce qui constitue une belle motivation pour décrypter la recette au préalable. Nous présentons ci-dessous le cadre de travail.

Tableau 1A - Organisation spatiale et support de travail (GS séance 2)

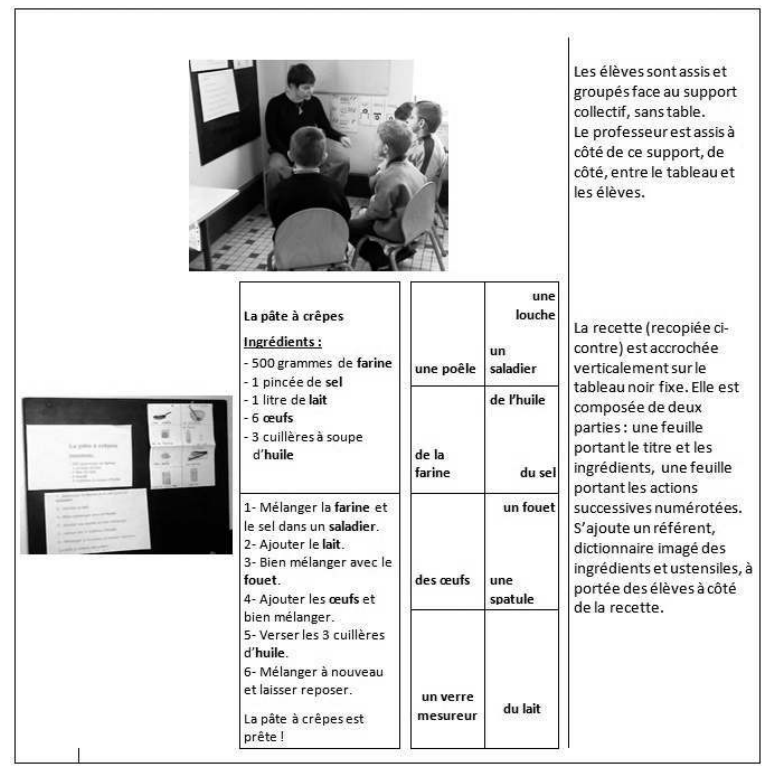

L'absence de tables est propice au déplacement des élèves, donc aux interventions - gestes ou tracés - des uns et des autres sur le support. La recette des crêpes est adaptée à l'intervention didactique collective : elle est agrandie de manière à ce que les élèves du petit groupe puissent la lire collectivement.

C'est le repérage visuel du mot qui est travaillé dans l'extrait qui va suivre. L'enjeu est de faire prendre conscience aux élèves de la présence de mots à l'écrit. L'apprentissage de cette compétence, qui s'inscrit en amont de l'identification des mots, est nécessaire, parce que le concept de mot au sens d'unité graphique n'est pas encore stable en fin de maternelle (Giasson, 2007).

Juste avant l'événement remarquable, un élève ${ }^{4}$ se déplace spontanément vers le support. L'enseignante reste assise mais se tourne vers ce même support. L'élève BA, désigne le dans huile comme un mot. L'enseignante se saisit de l'erreur de l'élève (le dans huile n'est pas un mot).

Tableau 1B - Mot le versus le dans huile

\begin{tabular}{|c|c|c|c|}
\hline Gestualité & Discours & Description de Paction & Fonction des gestes \\
\hline BA : un pointage sur le du mot huile & $\begin{array}{l}\text { BA : il y a le mot le là } \\
\text { en bas }\end{array}$ & $\begin{array}{l}\text { L'élève pointe avec } \\
\text { son index sur la fin } \\
\text { du mot huile. } \\
\text { Le professeur, resté } \\
\text { à sa place, approche } \\
\text { sa main du support. }\end{array}$ & $\begin{array}{l}\text { Ce pointage focalise } \\
\text { r'attention sur le de } \\
\text { huile. }\end{array}$ \\
\hline 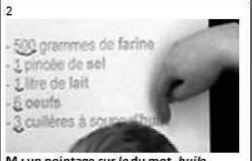 & $\begin{array}{l}\text { M: alors est-ce que } \\
\text { le mot le on peutle } \\
\mathrm{xxx}\end{array}$ & $\begin{array}{l}\text { Le professeur pointe } \\
\text { avecson index sur le } \\
\text { du mot huile. } \\
\text { BA et les autres } \\
\text { élèves regardentce } \\
\text { qui est désigné. }\end{array}$ & $\begin{array}{l}\text { Ce pointage focalise } \\
\text { l'attention sur le de } \\
\text { huile. }\end{array}$ \\
\hline 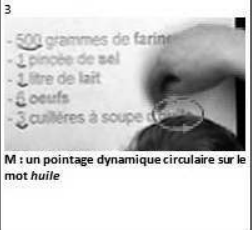 & $\begin{array}{l}\text { M : on l'a dans à } \\
\text { l'intérieur du mot? }\end{array}$ & $\begin{array}{l}\text { Le professeur pointe } \\
\text { sur le mot huile } \\
\text { qu'elle entoure avec } \\
\text { son index. } \\
\text { BA et les autres } \\
\text { élèves regardent ce } \\
\text { qui est désigné. }\end{array}$ & $\begin{array}{l}\text { Ces pointages sont } \\
\text { des enveloppes } \\
\text { gestuelles visuelles } \\
\text { dont la fonction est } \\
\text { de définir le mot } \\
\text { huile et le motle, } \\
\text { chacun comme des } \\
\text { unités graphiques à } \\
\text { mettre sur le même } \\
\text { plan. }\end{array}$ \\
\hline $\begin{array}{l}\text { M: un pointage dynamique circulaire sur le } \\
\text { mot-outil le }\end{array}$ & $\begin{array}{l}\text { M : regarde ici on a } \\
\text { le mot le il est tout } \\
\text { seulc'est un mot- } \\
\text { outil }\end{array}$ & $\begin{array}{l}\text { Le professeur pointe } \\
\text { sur le mot-outille } \\
\text { qu'elle entoure avec } \\
\text { son index. } \\
\text { LU s'approche. BA, } \\
\text { et les autres élèves } \\
\text { regardentce qui est } \\
\text { désigné. }\end{array}$ & \\
\hline espaces autour du mot-outilles & $\begin{array}{l}\text { M: il y a un trou } \\
\text { entre les mots }\end{array}$ & $\begin{array}{l}\text { Le professeur pointe } \\
\text { de deux doigts sur } \\
\text { les espaces autour } \\
\text { du mot-outille. } \\
\text { BA et les autres } \\
\text { élèves regardent ce } \\
\text { qui est désigné. }\end{array}$ & $\begin{array}{l}\text { Ces pointages } \\
\text { rappellent que les } \\
\text { unités graphiques } \\
\text { sont séparées par } \\
\text { des espaces. }\end{array}$ \\
\hline
\end{tabular}




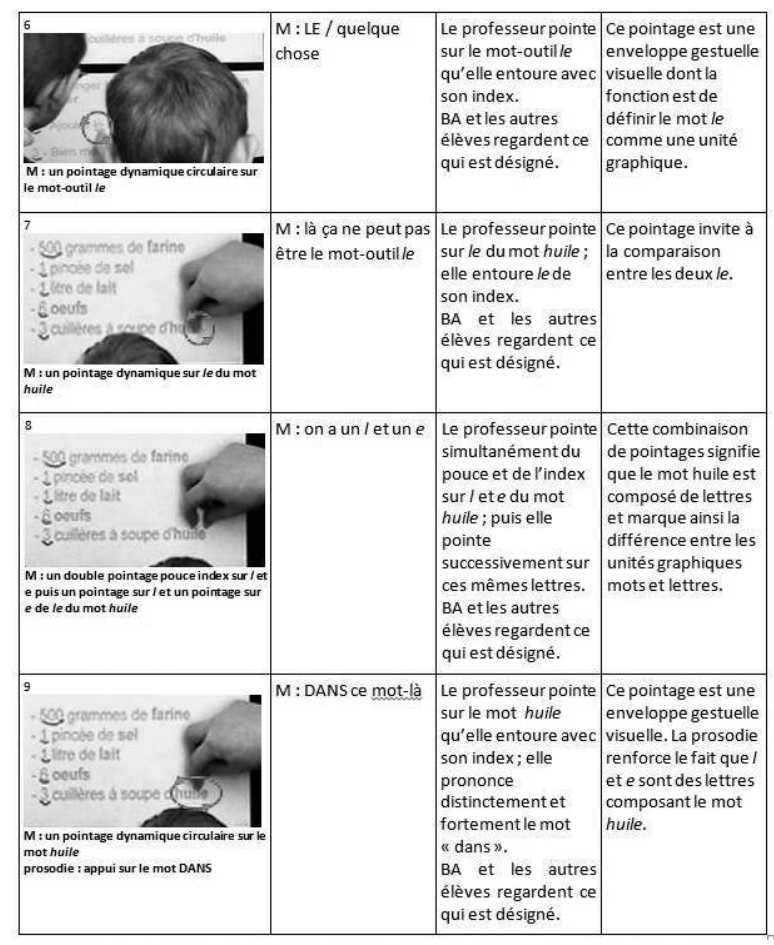

Il apparaît que l'enseignante déploie une gestualité dense et précise pour faire distinguer le groupe de lettres le à l'intérieur du mot huile et le mot le : des pointages simples pour focaliser l'attention; des pointages dynamiques circulaires, véritables enveloppes gestuelles autour des mots; des pointages dynamiques sur les espaces blancs qui délimitent les mots ; un double pointage sur les lettres ; une accentuation prosodique. Elle agit dans l'instant : ce qu'elle réalise avec précision est constitutif d'une pratique spécifique. L'usage de la matérialité extrinsèque ainsi mis au jour ne semble pas en lien avec une méthode.

\section{$2^{e}$ exemple : la matérialité comme ressource pour enseigner-apprendre la phonologie}

Cet événement remarquable est sélectionné sur la quatrième séance de Grande Section ( $4^{e}$ séance filmée de l'année scolaire et du recueil complet). En entretien semi-directif, l'enseignante explique qu'elle travaille la conscience phonologique dans des séances spécifiques, comme des gammes en musique. C'est l'objet de cette séance.

La conscience phonologique est définie comme « la capacité d'identifier les composantes phonologiques des unités linguistiques et de les manipuler de façon opérationnelle » (Gombert, 1992, in Giasson,
2007, p. 152). C'est donc une compétence métalinguistique qui consiste à réfléchir sur le langage en tant qu'objet, non de l'utiliser simplement comme outil de communication (ibid.). Dans le même sens, Vygotski (1934/1997, p. 376) explicite le problème de la conscience phonologique : «Bien que l'enfant utilise sans faute l'aspect phonétique de sa langue maternelle, il ne se rend pas compte des sons qu'il prononce. C'est pourquoi il a une grande peine, lorsqu'il écrit, à épeler le mot, à le décomposer en sons distincts ». Cette compétence, qui constitue une des clés de la maîtrise de la lecture puisqu'elle conditionne la connaissance du code phonographique, doit faire l'objet d'un apprentissage régulier pour être construite. L'enseignante ne dit pas autre chose lors du premier entretien semi-directif : « la conscience phonologique n'est pas quelque chose d'inné, elle se construit».

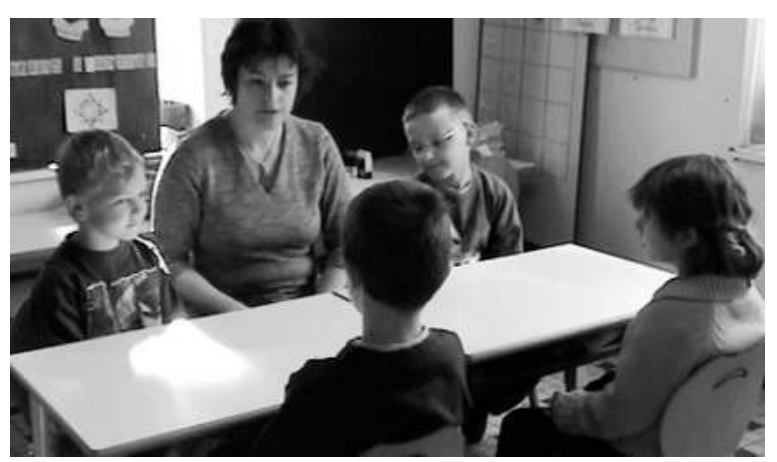

Figure 1 - Organisation spatiale (GS séance 4).

Les élèves et l'enseignante sont assis autour de deux tables accolées. LU et BA sont respectivement à gauche et à droite de l'enseignante; LO en face de BA et ÉL en face de LU. L'enseignante impose le silence et demande l'attention auditive et visuelle. 
Tableau 2 - La syllabe initiale

\begin{tabular}{|c|c|c|c|}
\hline Gestualité & Discours & Description de Paction & Fonction des gestes \\
\hline $\begin{array}{l}\text { M: } \\
\text { posture = garde ses mains } \\
\text { sousla table et regarde } \mathrm{BA}\end{array}$ & $\cdots$ & $\begin{array}{l}\text { Le professeurgarde } \\
\text { ses mains sous la } \\
\text { table, regarde } \\
\text { l'élève, ne bouge } \\
\text { pas, ne dit rien. } \\
\text { L'élève regarde } \\
\text { devant lui, prêt à } \\
\text { écouter. }\end{array}$ & $\begin{array}{l}\text { La posture est ici } \\
\text { gage de l'attention } \\
\text { demandée età } \\
\text { venir. } \\
\text { Le silence crée un } \\
\text { espace d'attente } \\
\text { attentive. }\end{array}$ \\
\hline $\begin{array}{l}\text { M: } \\
\text { angeste iconiquet } \\
\text { avec prosodie }=\end{array}$ & $\mathrm{M}: \mathrm{PA} /$ & $\begin{array}{l}\text { Le professeur pose } \\
\text { la main gauche } \\
\text { ouverte devant } \\
\text { l'élève en appuyant } \\
\text { fortement la } \\
\text { prononciation de la } \\
\text { syllabe " pa "; puis } \\
\text { elle marque une } \\
\text { courte coupure. } \\
\text { L'élève regarde le } \\
\text { geste et écoute. }\end{array}$ & \multirow[t]{2}{*}{$\begin{array}{l}\text { Chaque main posée } \\
\text { représente } \\
\text { visuellement une } \\
\text { syllabe prononcée. } \\
\\
\text { La prosodie permet } \\
\text { d'insister sur la } \\
\text { segmentation du } \\
\text { mot en deux } \\
\text { syllabes. }\end{array}$} \\
\hline $\begin{array}{l}\text { M: } \\
\text { un geste iconique } \\
\text { avee prosodie = } \\
\text { appui sur [pi] }\end{array}$ & $M: P I$ & $\begin{array}{l}\text { Le professeur pose } \\
\text { la main droite } \\
\text { ouverte devant } \\
\text { l'élève en appuyant } \\
\text { fortement la } \\
\text { prononciation de la } \\
\text { syllabe " pi ». } \\
\text { L'élève regarde le } \\
\text { geste et écoute. }\end{array}$ & \\
\hline $\begin{array}{l}\mathrm{M} \text { : } \\
\text { un geste iconique }\end{array}$ & $\begin{array}{l}\text { BA : PA } \\
M \text { : oui }\end{array}$ & $\begin{array}{l}\text { Le professeur replie } \\
\text { la main droite } \\
\text { ouverte devant } \\
\text { l'élève. } \\
\text { L'élève regarde le } \\
\text { geste et donne la } \\
\text { réponse. }\end{array}$ & $\begin{array}{l}\text { La main repliée } \\
\text { signifie : «on } \\
\text { cache la syllabe dans } \\
\text { la main ». }\end{array}$ \\
\hline
\end{tabular}

L'enseignante a pour objectif de faire prendre conscience de l'unité sonore syllabe du début d'un mot bisyllabique. Cet exercice est réalisé au moyen d'une « hyper-matérialisation » lisible sur le photogramme dans le contraste images denses/discours réduit. L'enseignante adopte une posture particulière au début et entre chaque exercice, regardant l'élève interrogé, posant ses mains sur ses genoux sous la table; puis elle attend un temps court en silence. Un espace d'attention est ainsi ouvert pour le groupe et pour le binôme enseignante/élève en particulier. Le son, presque immatériel parce qu'invisible, intouchable et fugace, est matérialisé d'une part par l'objet main, d'autre part par la prosodie.

En entretien d'auto-confrontation, l'enseignante explique qu'elle utilise un outil d'enseignementapprentissage de la phonologie : Conscience phonologique aux éditions La Cigale. L'exercice proposé ci-dessus est issu de cette méthode. Cependant, elle dit aussi : «Alors en fait cet exercice je l'ai adapté parce que normalement dans la méthode ils ne font pas visualiser les syllabes comme ça ils les font directement sans gestes [...] en fait les premières fois où je l'ai fait il y a des enfants qui n'arrivaient pas à cacher la première ou la deuxième syllabe donc en fait je les ai matérialisées ». Encore une fois l'enseignante n'est pas l'exécutante d'une méthode. Elle s'inspire de celle-ci mais elle adapte en permanence son enseignement à son public et aux situations. L'adaptation consiste ici en la matérialisation corporelle de la syllabe.

$3^{e}$ exemple : la matérialité comme ressource pour enseigner-apprendre le sens de l'écrit; le cas de la représentation mentale de la situation

Il s'agit de la quatrième séance du Cours Préparatoire ( $4^{\mathrm{e}}$ séance filmée de l'année scolaire et $8^{\mathrm{e}} \mathrm{du}$ recueil complet).

L'événement remarquable choisi est centré sur un extrait de l'album Mika et le jeteur de sorts constitutif de la méthode Mika. L'album est le support initial de lecture, il raconte les péripéties de l'histoire où l'héroïne, Mika, avec l'aide de Petit Oiseau, doit faire face au jeteur de sorts Maléfix. Mika est un personnage familier aux élèves, représentée par une marionnette trônant sur un meuble de la classe.

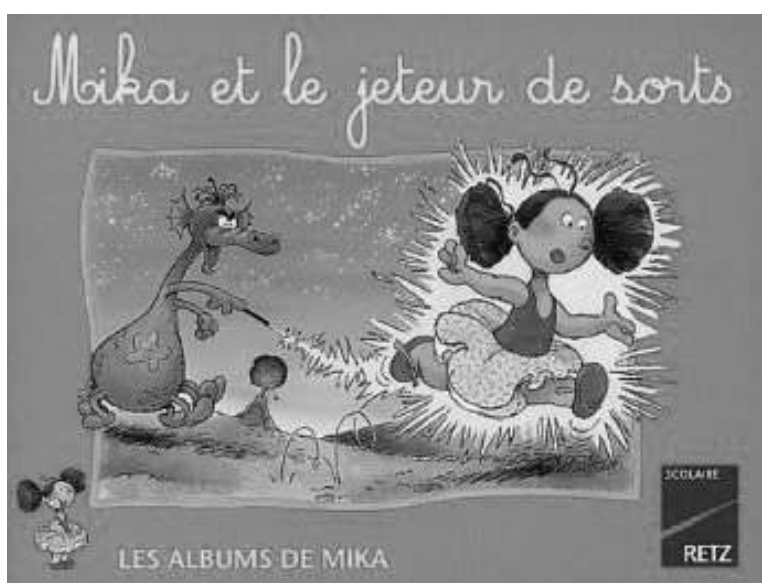

Figure 2 - Support initial de la $4^{\mathrm{e}}$ séance de Cours Préparatoire : l'album Mika et le jeteur de sort.

L'enseignante a créé un support dérivé de l'album : une grande affiche sur laquelle le texte de l'extrait a été recopié en gros caractères pour une exploitation collective. Voici ci-après l'organisation de classe pour l'événement remarquable sélectionné. 
Tableau 3A - Organisation spatiale et support de travail (CP début séance 4)

\begin{tabular}{|c|c|c|}
\hline & & $\begin{array}{l}\text { Les élèves sont assis à } \\
\text { leur table face au } \\
\text { professeur et au } \\
\text { support. } \\
\text { Le professeur est } \\
\text { debout entre le tableau } \\
\text { et les élèves, la } \\
\text { baguette } \\
\text { La à la main. } \\
\text { éuille sur la table } \\
\text { est sans lien avec } \\
\text { l'activité en cours. }\end{array}$ \\
\hline 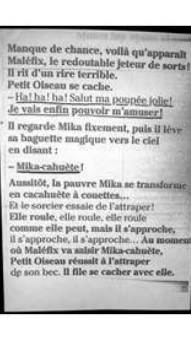 & $\begin{array}{l}\text { Manque de chance, voilà qu'apparait } \\
\text { Maléfix, le redoutable jeteur de sorts ! II rit } \\
\text { d'un rire terrible. Petit Oiseau se cache. } \\
\text { - Ha ! ha ! ha ! Salut ma poupée jolie ! Je } \\
\text { vais enfin pouvoir m'amuser ! } \\
\text { II regarde Mika fixement, puis il lève sa } \\
\text { baguette magique vers le ciel en disant : } \\
\text { - Mika-cahuète ! } \\
\text { Aussitôt, la paurre Mika se transforme en } \\
\text { cacahuete à couettes... Et le sorcier essaie } \\
\text { de ''attraper! Elle roule, elle roule, elle } \\
\text { roule comme elle peut, mais il s'approche, } \\
\text { il s'approche, il s'approche... Au moment } \\
\text { où Maléfix va saisir Mika-cahuète, Petit } \\
\text { Oiseau réussit à l'attraper de son bec. II file } \\
\text { se cacher avec elle. }\end{array}$ & $\begin{array}{l}\text { Le texte, en grand } \\
\text { format (recopié ci- } \\
\text { contre), est accroché } \\
\text { verticalement surle } \\
\text { tableau noir. }\end{array}$ \\
\hline
\end{tabular}

L'extrait a été déchiffré antérieurement par le groupe lors de la séance précédente. L'identification des mots n'étant pas parfaitement automatisée, cette activité de déchiffrage sollicite encore toute l'attention du lecteur en apprentissage, ce qui ne lui permet pas d'être disponible pour comprendre de manière exhaustive sur le champ. En outre, Goigoux (cf. supra) défend l'idée que la compréhension ne se donne pas automatiquement après le déchiffrage : comprendre s'apprend. L'enseignante s'inscrit dans cette conception, elle revient donc sur l'extrait pour mener un travail d'enseignement de sa compréhension.
Tableau 3B - La représentation mentale

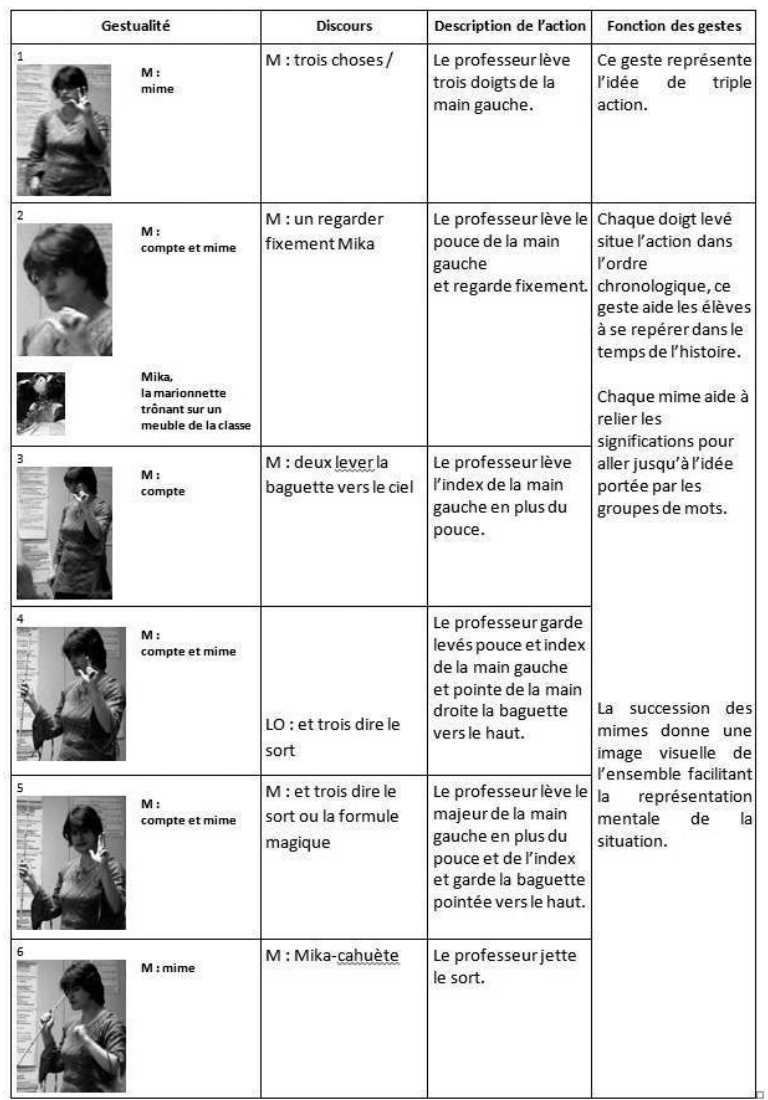

La gestualité déployée au fil de l'événement sert la construction de la représentation mentale

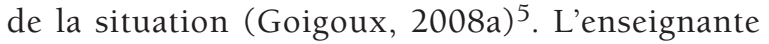
théâtralise, mime ce qui vient d'être lu au moyen des objets impliqués dans l'extrait, donne vie à la scène. Elle procède par étapes en comptant sur ses doigts les actions engagées pour jeter le sort et en les vivant une à une. Elle « fait le film» de bout en bout, les élèves regardent le film. Il est donc question du sens de l'histoire pour des actions décrites de manière précise et explicite. Ces actions sont rendues visibles par le mime incluant les objets impliqués. Le mime apparaît comme un moyen matériel extrinsèque de faire accéder les élèves au sens qui, par sa nature symbolique, ne présente pas la même matérialité intrinsèque que l'écrit codé sur le papier. En entretien d'auto-confrontation, l'enseignante dit avoir fait mimer aussi les scènes de combat quand Mika terrasse le jeteur de sorts. Le sens est incarné, " corporéisé », matérialisé directement dans l'action elle-même. 
$4^{e}$ exemple : la matérialité comme ressource pour enseigner-apprendre les codes écrits ; le cas du code morphographique

L'événement remarquable dont il est question ici est issu de la même séance. L'agencement spatial et le support sont cependant différents :

Tableau 4A - Organisation spatiale et support de travail (CP fin séance 4)

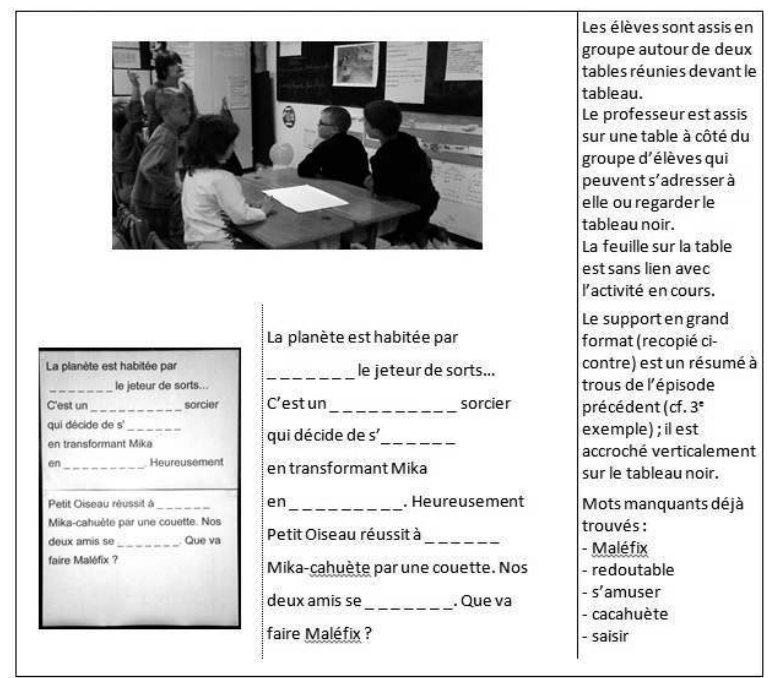

Les élèves ont pour tâche collective d'écrire les mots manquants dans le résumé du texte supra. L'exercice demande un effort de compréhension puisque les phrases doivent avoir du sens et, qui plus est, respecter la signification de l'épisode initial. Rappelons ici que l'écriture est considérée comme plus active que la lecture, permet de prendre conscience des caractéristiques de l'écrit et d'acquérir les correspondances phonographiques naturellement (Fijalkow, 2003).

Lors de cet événement remarquable, l'activité vise à faire apprendre le code morphographique (Chauveau, 1998), en l'occurrence le morphogramme grammatical -nt. Pour trouver le mot manquant, les élèves s'appuient sur les tirets tracés préalablement par l'enseignante : un tiret représente une lettre. C'est la fin de l'exercice, il ne reste qu'un mot à trouver : cachent dans la phrase Nos deux amis se cachent. Juste avant cet événement, les élèves ont trouvé que le mot manquant était cachent. Cependant ils comptaient écrire cache. Ils ont constaté que le nombre de lettres à inscrire différait du nombre de tirets. L'enseignante leur a dit qu'ils avaient un problème à résoudre sachant que le mot à écrire était bien cache (nt).
Tableau 4B - Le pluriel s versus nt

\begin{tabular}{|c|c|c|c|}
\hline Gestualité & Discours & Description de Paction & Fonction des gestes \\
\hline$\frac{1}{\text { Absence de gestes }}$ & $\begin{array}{l}\text { LO : benes } \\
M: \text { alors pourquoi } \\
\text { tu voudrais mettre } e \\
s ? \\
\text { LO : ben parce qu'ils } \\
\text { sont et bene } s \text { enfin } \\
\text { le } s \text { il veut dire } \\
\text { plusieurs } \\
\text { M : le } s \text { voudrait dire } \\
\text { plusieurs alors je } \\
\text { vais donnerun } \\
\text { indice } \\
\text { supplémentaire } \\
\text { BA : le } x \text { aussiil veut } \\
\text { dire plusieurs LO }\end{array}$ & $\begin{array}{l}\text { Les élèves font des } \\
\text { propositions pour } \\
\text { compléter la fin du } \\
\text { mot cache__; is } \\
\text { regardent tour à } \\
\text { tour le support et le } \\
\text { professeur. } \\
\text { Celui-ci pose des } \\
\text { questions pour } \\
\text { orienter la réflexion } \\
\text { et régule lactivité. }\end{array}$ & $\begin{array}{l}\text { Le temps sans geste } \\
\text { laisse toute la place } \\
\text { à la réflexion et à la } \\
\text { discussion. } \\
\text { dis. }\end{array}$ \\
\hline$\underbrace{------1}_{\mathrm{M}: \text { tracé rouge sous le mot àecrire }}$ & $\begin{array}{l}\text { M : je vais donner } \\
\text { un indice } \\
\text { supplémentaire en } \\
\text { vous soulignant ce } \\
\text { mot } \\
\text { Éls : ah c'est un mot } \\
\text { rouge }\end{array}$ & $\begin{array}{l}\text { Le professeur } \\
\text { souligne en rouge } \\
\text { les tirets } \\
\text { représentant les } \\
\text { lettres du motà } \\
\text { écrire. } \\
\text { Les élèves regardent } \\
\text { et réagissenten } \\
\text { s'exclamant. }\end{array}$ & $\begin{array}{l}\text { Le soulignementen } \\
\text { rouge indique que le } \\
\text { mot à trouver est un } \\
\text { « mot rouge ", } \\
\text { terminologie } \\
\text { écologique utilisée } \\
\text { en lieu et place de la } \\
\text { terminologie } \\
\text { universelle } \\
\text { " verbe ". } \\
\text { L'exclamation } \\
\text { traduit le fait que la } \\
\text { couleur rouge } \\
\text { " parle " aux élèves. }\end{array}$ \\
\hline
\end{tabular}

L'enseignante donne donc un indice fort en traçant un trait rouge, à considérer comme constitutif de la matérialité extrinsèque objectale. Lors de l'entretien d'auto-confrontation, elle dit employer un code couleur pour faire de la grammaire implicite. Peu à peu il y a explicitation puisque la marque du pluriel des verbes à la troisième personne du pluriel est repérée. Elle parle d'étapes d'apprentissage qui mènent à la connaissance du verbe grâce à des petits signaux visuels supposés stimuler la mémoire des élèves.

Le code couleur est écologique et spécifique à la façon de procéder de l'enseignante. Il fait certes penser à des systèmes multimodaux d'apprentissage de l'écrit ${ }^{6}$; cependant, l'enseignante ne revendique aucune méthode. Elle utilise son propre code matériel, élaboré dans la classe particulière qui est la sienne.

\section{$5^{e}$ exemple : la matérialité comme ressource pour enseigner-apprendre le sens de l'écrit; le cas du lexique}

Le thème de cette deuxième séance de Cours Élémentaire $1^{\text {re }}$ année ( $2^{\mathrm{e}}$ séance filmée de l'année scolaire et $10^{\mathrm{e}} \mathrm{du}$ recueil complet) est la lecture de documentaires sur les marionnettes Polichinelle et Guignol. Voici comment est agencé l'espace pour cette séance : 
Tableau 5A - Organisation spatiale et support de travail (CEl séance 2 )

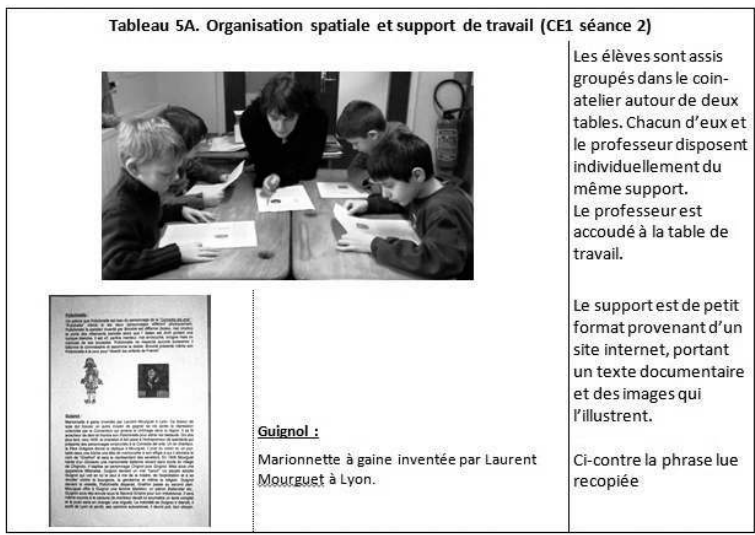

Les supports collectifs dérivés laissent place ici à un support initial i.e. qui n'a pas subi de transformation. De plus chaque élève dispose de sa feuille personnelle qu'il lit en même temps que ses camarades. Parce que les élèves sont devenus lecteurs, ils sont capables de suivre la leçon de lecture à partir d'un support individuel et de petit format.

La connaissance du vocabulaire contenu dans un texte conditionne sa compréhension (Giasson, 2007). La lecture est, entre autres, une activité linguistique engageant les connaissances lexicales et syntaxiques (Goigoux, 2008a). En effet, même si l'élève est capable de déchiffrer un mot écrit, si cependant il n'en connaît pas la signification, la compréhension d'ensemble est altérée. C'est pourquoi l'accroissement du lexique au fil de la scolarité est essentiel. L'événement remarquable qui suit donne à voir un travail sur le lexique plus précisément sur les différents types de marionnettes. La phrase « Marionnette à gaine inventée par Laurent Mourguet à Lyon» vient d'être lue par l'élève LA. L'enseignante lance une question sur le sens de l'expression « marionnette à gaine ».
Tableau 5B - Le lexique : les différents types de marionnettes

\begin{tabular}{|c|c|c|c|}
\hline Gestualité & Discours & Description de Paction & Fonction des gestes \\
\hline Absence de gestes & $\begin{array}{l}M: c^{\prime} \text { est quoi une } \\
\text { marionnette à } \\
\text { gaine? } \\
\text { LO: à cordes? }\end{array}$ & $\begin{array}{l}\text { Le professeur } \\
\text { questionne. } \\
\text { Les élèves } \\
\text { réfléchissent. } \\
\text { L'élève LO tente une } \\
\text { réponse. }\end{array}$ & \\
\hline M : geste iconi & $\begin{array}{l}\text { M: non } \\
\text { LO:à fils }\end{array}$ & $\begin{array}{l}\text { Le professeur } \\
\text { effectue ungeste } \\
\text { iconique dynamique } \\
\text { représentant un fil. } \\
\text { Les élèves le } \\
\text { regardent. } \\
\text { L'élève LO propose } \\
\text { une autre réponse. }\end{array}$ & $\begin{array}{l}\text { Ce geste a pour } \\
\text { fonction de donner } \\
\text { une représentation } \\
\text { du fil. }\end{array}$ \\
\hline & $\begin{array}{l}\text { M : à fils c'est avec } \\
\text { des fils }\end{array}$ & $\begin{array}{l}\text { Le professeur mime } \\
\text { le mouvement } \\
\text { appliqué aux fils. } \\
\text { Les élèves le } \\
\text { regardent. }\end{array}$ & $\begin{array}{l}\text { Ce geste a pour } \\
\text { fonction de donner } \\
\text { une représentation } \\
\text { de la manière dont } \\
\text { on actionne une } \\
\text { marionnette à fils. }\end{array}$ \\
\hline & $\begin{array}{l}\text { M : mais à gaine } \\
\text { vous en connaissez } \\
\text { une de marionnette } \\
\text { à gaine }\end{array}$ & $\begin{array}{l}\text { Le professeur } \\
\text { questionne. } \\
\text { Les élèves } \\
\text { réfléchissent. }\end{array}$ & $\begin{array}{l}\text { Ces deux gestes ont } \\
\text { pour fonction de } \\
\text { montrer que la main } \\
\text { est sollicitée pour } \\
\text { actionner la } \\
\text { marionnette à gaine. }\end{array}$ \\
\hline $\begin{array}{l}\text { Él:mime }= \\
\text { deux gestes iconiques }\end{array}$ & $\begin{array}{l}\text { Él : ah Mika } ~ \\
\text { M : Mika est une } \\
\text { marionnette à gaine }\end{array}$ & $\begin{array}{l}\text { L'élève ÉL effectue } \\
\text { deux gestes } \\
\text { iconiques } \\
\text { dynamiques } \\
\text { représentantle } \\
\text { mouvement de la } \\
\text { main appliqué à une } \\
\text { marionnette à gaine. } \\
\text { Les autres élèves et } \\
\text { le professeur la } \\
\text { regardent. }\end{array}$ & \\
\hline
\end{tabular}

L'enseignante poursuivra longuement sur les marottes : elle se saisira du crayon sur la table pour représenter le bâton et elle effectuera un mouvement circulaire au-dessus de ce crayon debout pour représenter la tête de la marotte...

La mimogestualité apparaît donc comme une ressource pour enseigner le lexique. À l'instar du Geste Pédagogique (GP) pour l'enseignement de la langue étrangère (Tellier, 2008, 2010), le mime facilite l'accès au sens en traduisant gestuellement les paroles de l'enseignant. Il donne représentations concrètes des objets.

Colletta (2004b) montre que les enfants ont à leur disposition tout un répertoire coverbal (l'élève ÉL mime aussi). Ces gestes accompagnent le discours verbal et ne s'appliquent pas à l'objet. Le mime est utilisé ici pour faire comprendre le vocabulaire nouveau. L'enseignement de la compréhension au moyen de ces mimes ne peut advenir que parce qu'ils sont partagés de sens commun avec l'adulte. Colletta (p. 60) affirme en effet que « la posturo-mimogestualité communicative des enfants scolarisés à l'école élémentaire ne diffère pas fondamentalement de celle 
des adultes ». Les coverbaux sont donc détournés à des fins didactiques. En conséquence, ils s'appliquent à l'objet de savoir qui est le sens du texte lu. Ils constituent des gestes de sens épistémiques.

\section{POUR CONCLURE}

La matérialité extrinsèque est polymorphe et systémique, associant des objets pour la plupart traditionnels dans une classe, et des agents corporels composites, incluant aussi d'autres paramètres matériels tels la proxémie et l'agencement spatial. Pourquoi l'enseignante recourt-elle à la matérialité ? Ni la matérialité intrinsèque de l'écrit (codes alphabétiques et extra-alphabétiques) ni le sens de l'écrit ne sont accessibles à l'élève non lecteur en apprentissage. De ce fait, l'enseignante doit montrer, pointer, souligner, annoter matériellement l'écrit d'une part et, d'autre part, produire des représentations intermédiaires matérielles des significations inaccessibles en l'état. Proposer aux élèves d'apprendre un savoir au-delà, i.e. en rupture avec leur déjà-là, oblige en réalité à tisser les fils de l'existant et de l'à-venir. La matérialité apparaît comme une ressource liant le connu à l'inconnu.

Des systèmes sémiotiques variés et spécialisés sont donc à l'œuvre pour amener l'élève à l'appropriation de la lecture : les exemples ci-dessus donnent à voir les pointages divers et les tracés pour les codes, les gestes et la prosodie en phonologie, la mimogestualité pour l'élucidation du sens. Il apparaît une adaptation continue entre matérialité extrinsèque et matérialité intrinsèque. Par exemple les pointages travaillés (extrinsèque) permettent de contraindre l'attention des élèves et de faire appréhender le mot (intrinsèque) en le délimitant, en l'enveloppant, en l'isolant. Matérialité extrinsèque et matérialité intrinsèque dialoguent au gré des visées enseignantes qui se dessinent et évoluent au fil de l'avancée didactique. La première "épouse » la seconde mais se transforme en fonction des besoins d'apprentissage qui se font jour progressivement. Le dialogue extrinsèque/intrinsèque n'est pas écrit d'avance, il prend corps dans l'action didactique conjointe (Sensevy $\&$ Mercier, 2007).

Cet usage de la matérialité extrinsèque dans l'activité est personnel et se développe au fil du temps dans l'exercice du métier. L'enseignante en parle clairement en entretien d'auto-confrontation. À la ques- tion : «Ces gestes-là est-ce qu'on vous les a appris ? » elle répond : «Ce n'est pas ça l'IUFM ${ }^{\top}$ qu'on nous en a vraiment parlé $[\ldots]$ je suis toujours en recherche de façons de faire mémoriser ou de faire apprendre [...] la maternelle m'a apporté beaucoup je pense qu'il $\mathrm{y}$ a des façons de fonctionner qu'on devrait utiliser jusqu'au cycle $3 »$. C'est l'expérience professionnelle qui la mène à l'exercice aiguisé de la matérialité dans les situations d'enseignement-apprentissage. Il s'ensuit que la méthode de lecture, en l'occurrence Mika, est incarnée par l'enseignante dont les actions, au-delà de la méthode choisie, apparaissent comme complexes, précises mais surtout personnalisées.

L'efficacité de l'enseignement réside dans l'« art » de faire construire les connaissances par l'élève. La réticence didactique (Sensevy \& Quilio, 2002) est une des clés de cette efficacité : dire sans dire, faire percevoir et discerner en évoquant et suggérant. La matérialité extrinsèque outille la réticence didactique. En créant un premier lien avec la nouveauté, mais sans la dévoiler directement, elle a la fonction capitale d'agent bâtisseur des connaissances de l'élève.

\section{NOTES}

1. Selon Vygotski, le développement psychologique (formation des fonctions psychiques supérieures) se déroule en deux temps : le ler développement correspond au temps ponctuel d'enseignement-apprentissage de nouvelles notions guidé par l'enseignant ; le $2^{\mathrm{e}}$ développement correspond à la réorganisation interne, à plus ou moins long terme, de l'ensemble des connaissances de l'élève.

2. La proxémie correspond à la distance physique qui s'établit entre deux personnes prises dans une interaction (Hall, 1066/1971).

3. Le support devient progressivement individuel au Cours Élémentaire lre année.

4. Dans la suite du texte, les élèves sont désignés par BA, LO, LA, ÉL, CO ; les élèves ensemble par Éls ; l'enseignante par $\mathrm{M}$.

5. Rappelons que comprendre la situation évoquée par le texte suppose de relier les significations portées par les groupes de mots et de construire la représentation mentale ( «le film») de cette situation.

6. Par exemple la méthode Aloé de Brigitte Roy, MarieOdile Martin et Marie Staebler, éditée par Commédic, Nancy; ou encore la lecture en couleurs de Gattegno (cf. le site www.uneeducation pourdemain.org).

7. Institut Universitaire de la Formation des Maîtres 


\section{RÉFÉRENCES}

Allègre, C. (2007). 10+1 questions à Claude Allègre. Courtry : Michallon.

Blanchet, A., \& Gotman, A. (2001). L'enquête et ses méthodes : l'entretien. Paris : Nathan/VUEF.

Blaser, C. (2009). Le synopsis : un outil méthodologique pour comprendre la pratique enseignante. Nouveaux cahiers de la recherche en éducation, XII (1), p. 117-129.

Catach, N. (1978/2003). Lorthographe. Paris : PUF Que sais-je?

Céfaï, D. (2010). L'engagement ethnographique. Paris : EHESS.

Chauveau, G. (1998). Comment l'enfant devient lecteur. Paris : Retz.

Colletta, J.-M. (2004a). Communication non verbale et parole multimodale : quelles implications didactiques ? Le français dans le monde, 151-153.

Colletta, J.-M. (2004b). Le développement de la parole chez l'enfant âgé de 6 à 11 ans. Sprimont : Mardaga.

Dehaene, S. (2007). Les neurones de la lecture. Paris : Odile Jacob.

Dimitrova, N. (2012, mai 8). Développement de la communication intentionnelle gestuelle à partir des usages culturels des objets dans l'interaction triadique enfant-objet-adulte. Thèse de doctorat sous la direction de C. Moro. Lausanne : UNIL.

Fijalkow, J. (2003). Pourquoi et comment articuler l'apprentissage de la lecture avec celui de la production de d'écrit aux différentes étapes de la scolarité primaire? université de Toulouse le Mirail : EURED CREFI.

Forest, D. (2006). Analyse proxémique d'interactions didactiques. Carrefours de l'éducation (21), p. 73-94.

Giasson, J. (2005/2007). La lecture. De la théorie à la pratique. Bruxelles: De Boeck.

Goigoux, R. (2002). Analyser l'activité d'enseignement de la lecture : une monographie. Revue française de pédagogie (138), p. 125-134.

Goigoux, R. (2003). Réponse de Roland Goigoux à la seconde question de la conférence de consensus. Document envoyé au PIREF en vue de la conférence de consensus sur l'enseignement de la lecture à l'école primaire les 4 et 5 décembre 2003, Piref.

Goigoux, R. (2008a, mars). Apprendre à lire entre 4 et 8 ans. Montage vidéo de 5 h réalisé par SEM productions à l'issue d'un cycle de conférences pour la direction de l'enseignement public du Canton et de la République de Genève en mars 2008. Disponible en DVD et sur le web. http://wwwedu.ge.ch/sem/production/streaming/goigoux/goigoux_1.asp et_2.asp.

Goigoux, R. (2008b). Méthodes de lecture. In A. Van Zanten, Dictionnaire de l'éducation (p. 444-446). Paris : PUF Quadrige.

Hall, E. T. (1966/1971). La dimension cachée. Paris : Le Seuil.

Ministère de l'Éducation nationale. (2002). Qu'apprend-on à l'école maternelle ? Les nouveaux programmes. Paris : CNDP-XO Éditions.
Ministère de l'Éducation nationale. (2006a). Qu'apprendon à l'école élémentaire ? 2006-2007 Les programmes. Paris: CNDP-XO Éditions.

Ministère de l'Éducation nationale. (2006b, mai). Apprendre à lire. Guide thématique, Direction de l'enseignement scolaire Inspection générale de l'éducation nationale, Délégation à la communication.

Ministère de l'Éducation nationale. (2008, juin 19). Horaires et programmes d'enseignement de l'école primaire. B.O. $\mathrm{n}^{\circ} 3$ hors-série. Paris : SCÉRÉN-CNDP.

Moro, C., \& Rodríguez, C. (2005). Lobjet et la construction de son usage chez le bébé. Une approche sémiotique du développement préverbal. Berne : Peter Lang.

Moro, C. (2013). La notion de situation pour appréhender le développement de la parole à l'école maternelle française : les situations du «Panoptique » et de l'« Élève Blessé » comme exemples. In J.-P. Bernié, \& M. Brossard (Éds), Vygotski et l'école : apports et limites d'un modèle théorique pour penser l'éducation et la formation (p. 142-153). Bordeaux : Presses Universitaires de Bordeaux.

Moro, C., \& Joannes, É. (à paraître). Development of Speech through Materiality in French Nursery School. A Case Study at $2 \mathrm{nd} / 3 \mathrm{rd} \& 5$ th/6th years old during Activities based on the Story of « The Three Little Pigs $»$.

Nonnon, É., \& Goigoux, R. (2008). Travail de l'enseignant, travail de l'élève dans l'apprentissage initial de la lecture. Repères (36/2007).

Sensevy, G. (2011). Comprendre l'action didactique : méthode et jeux d'échelle. Consulté le 2 mars 2014, sur http://www.python.espe-bretagne.fr/sensevy/sens du savoir/

Sensevy, G., \& Mercier, A. (2007). Agir ensemble. L'action didactique conjointe du professeur et des élèves. Rennes : PUR.

Sensevy, G., \& Quilio, S. (2002). Les discours du professeur : vers une pragmatique didactique. Revue française de pédagogie, $\mathrm{n}^{\circ} 141,47-56$.

Taisson-Perdicakis, C. (2013). La situation d'enseignement-apprentissage de la lecture en grande section : un reflet de la zone proximale de développement dans ses tensions entre matérialités. In J.-P. Bernié, \& M. Brossard (Éds), Vygotski et l'école : apports et limites d'un modèle théorique pour penser l'éducation et la formation (p. 172-189). Bordeaux : Presses Universitaires de Bordeaux.

Tapparel, S. (2013). Micro-rupture et développement psychologique en situation éducative dans un centre de vie enfantine. Lobjet « brosse à dent» comme outil pour peindre en situation peinture. In J.-P. Bernié, \& M. Brossard (Éds), Vygotski et l'école : apports et limites d'un modèle théorique pour penser l'éducation et la formation (p. 136-141). Bordeaux : Presses Universitaires de Bordeaux.

Tellier, M. (2008). Dire avec des gestes. In F. Chnane-Davin, \& J.-P. Cuq (Éds.), Du discours de l'enseignant aux pratiques de l'apprenant en classe de français langue 
étrangère, seconde et maternelle (vol. 44). Recherches et Applications/Le Français dans le Monde.

Tellier, M. (2010). Faire un geste pour l'apprentissage : Le geste pédagogique dans l'enseignement précoce. In J. Sauvage, \& C. Corblin (Éds.), L'apprentissage des langues vivantes à l'école. Impacts sur le développement de la langue maternelle (éd. Enfance \& Langages, p. 31-54). Paris : L'Harmattan.

Vygotski, L. S. (1933/2012a). Analyse paidologique du processus pédagogique. In F. Yvon, \& Y. Zinchenko, Vygotski, une théorie du développement et de l'éducation. Recueil de textes et commentaires (p. 141-171). Moscou lomonossov: Faculté de psychologie de l'Université d'État.

Vygotski, L. S. (1933/2012b). La dynamique du développement intellectuel de l'enfant en lien avec l'enseignement. In F. Yvon, \& Y. Zinchenko, Vygotski, une théorie du développement et de l'éducation Recueil de textes et commentaires (p. 172-204). Moscou lomonossov : Faculté de psychologie de l'Université d'État.

Vygotski, L. S. (1934/1997). Pensée et langage. Paris : La Dispute.

Vygotski, L. S. (1935/1985). Le problème de l'enseignement et du développement à l'âge scolaire. In B. Schneuwly, \& J.-P. Bronckart, Vygotski aujourd'hui (p. 95-117). Neuchâtel - Paris : Delachaux \& Niestlé.

Ziegler, J. C. (2008). Développement de la lecture. In A. Van Zanten, Dictionnaire de l'éducation (p. 440-444). Paris : PUF Quadrige. 